\title{
Nueva propuesta para la gestión del conocimiento en la educación superior desde la andragogía creativa: Estudio de caso
}

\section{New proposal for the management of knowledge in higher education from the creative andragogy: Case study}

DOI: https://doi.org/10.17981/bilo.01.01.2019.02

Fecha de Recepción: 12/11/2019. Fecha de Aceptación: 01/12/2019. Fecha de Publicación: 11/12/2019

\author{
Tatiana Barrios-Arrieta \\ Universidad de la Costa CUC. Barranquilla, (Colombia) \\ tarrieta1@cuc.edu.co \\ Claudia Medina-Mosquera \\ Universidad de la Costa CUC. Barranquilla, (Colombia) \\ cmedina4@cuc.edu.co \\ Andrés Guarín- García \\ Universidad de la Costa CUC. Barranquilla, (Colombia) \\ aguarin@cuc.edu.co \\ Javier Carpintero-Durango \\ Universidad de la Costa CUC. Barranquilla, (Colombia) \\ jcarpint3@cuc.edu.co
}

\section{Resumen}

Esta investigación empleó aulas de clases para validar una serie de técnicas y herramientas que guían al estudiante a crear sus propias propuestas de aprendizaje desde la propuesta metodológica de Gamificación, neurociencia y pedagogía lúdica. Los resultados alcanzados en este escenario sugieren que desde la promoción de la autonomía del alumno, se abren mayores posibilidades para alcanzar objetivos e indicadores del plan de asignatura, confirmando que la Gestión del Conocimiento es un proceso de innovación abier ta con una retroalimentación constante del Capital Humano.

Palabras clave

Neurociencias, andragogía, gamificación, pedagogía lúdica

\section{Abstract}

This research used classrooms to validate a series of techniques and tools that guide the student to create their own learning proposals from the methodological proposal of Gamification, neuroscience and playful pedagogy. The results achieved in this scenario suggest that from the promotion of student autonomy, greater possibilities open up to achieve objectives and indicators of the subject plan, confirming that Knowledge Management is an open innovation process with constant feedback from Human Capital.

\section{Key Words}

Neuro sciences, Andragogy, gamification, ludic pedagogy 


\section{INTRODUCCIÓN}

La gestión del conocimiento como proceso para desarrollar innovación en las organizaciones, en la actualidad se consolida como el plan estratégico para obtener resultados en el desarrollo del capital humano con el objetivo de maximizar habilidades del saber ser, saber saber y saber hacer, llevando a las personas a ser más competitivas. Este concepto empleado en culturas empresariales y basado en comunicar la experiencia, la información y los conocimientos individuales y colectivos del grupo, tiene el potencial de implementarse en aulas de clase de la educación superior para promover en los futuros profesionales, las aptitudes asociadas al aumento de la productividad de un grupo de personas en un proceso productivo y visualizarlas como el principal activo de las organizaciones [1]. Sin embargo, un desafío en este proceso está en cómo se puede adaptar este concepto de cultura empresarial mientras que al mismo tiempo se incluyen técnicas que promuevan mejoras a la enseñanza por competencia en estos escenarios de formación profesional [2]. Se cree que una posible alternativa está con la implementación de estrategias pedagógicas como la gamificación o con la andragogía, ya que se puede renovar la enseñanza y empoderar a los alumnos a que de forma autónoma y explotaría [3] logren apropiarse de los conocimientos que los pueden llevar a maximizar sus aptitudes del saber ser, saber saber y saber hacer.

En el caso de la Gamificación, se aplican las metáforas de los juegos a las tareas de la vida real, para influir en el comportamiento del alumno y mejorar la motivación para la resolución de un problema y a la vez se aportan diferentes maneras de aprendizaje [4], [5], [6]. No obstante, el desafío de esta iniciativa está en cómo aplicarla en una población heterogénea que involucra a población adolescente (adulto Joven) y adulta, típica de los espacios universitarios. Es así como se propone hacer uso del conjunto de técnicas asociadas a la andragogía, el cual es definida como el arte y la ciencia de ayudar a adultos a aprender [7]. Con la andragogía se piensa que los adultos y adultos jóvenes necesitan ser participantes activos en su propio aprendizaje, manifestando que ellos aprenden de manera diferente a los niños y que los entrenadores en su rol de facilitadores del aprendizaje deberían usar un proceso diferente. Esta investigación propone una novedosa metodología llamada "andragogía creativa". La metodología propuesta "andragogía creativa" está apoyada en la neurociencia dado que agrupa a diferentes disciplinas que tienen como compromiso básico conocer el funcionamiento del cerebro teniendo en cuenta la individualidad de cada participante, en el desarrollo y búsqueda de diferentes formas de aprender [8], [9].

El estudio de caso para la validación de esta investigación fue en alumnos de pregrado del programa de ingeniería industrial de la Universidad de la Costa, Colombia. Se espera que con estos resultados se creen alternativas que faciliten el proceso de enseñanza por competencias bajo una visión divergente y real encaminada al sector empresarial (Torres, 2009).

\section{METODOLOGÍA}

La siguiente metodología se encuentra descrita en la figura 1. Como estudio de caso, se valida esta propuesta en las aulas de clases de la Universidad de la Costa, de la facultad de Ingeniería Industrial y la toma de datos es a través de encuestas, con el fin de medir las expectativas del estudiante ante el desarrollo de las clases dentro de su programa de formación, buscando hacerlo partícipe en el diseño de las clases y eliminando el modo tradicional o magistral.

Considerando que la población del programa de pregrado de ingeniería industrial de esta institución es cercana 1369 alumnos (CUC, 2018), se propone un nivel de confianza del 90\% y asimismo un error de estimación máximo del 10\%. Se emplea la siguiente formulación para calcular el tamaño de la muestra para esta población finita considerando un 50\% de probabilidad de que ocurra el evento estudiado como se muestra en la ecuación (1):

$$
\mathrm{n}=\frac{\mathrm{N} \times \mathrm{Z}_{\mathrm{a}}^{2} \times \mathrm{p} \times \mathrm{q}}{\left\{\mathrm{e}^{2} \times(\mathrm{N}-1)\right\}+\left(\mathrm{Z}_{\mathrm{a}}^{2} \times \mathrm{p} \times \mathrm{q}\right)}
$$

Donde:

n: Tamaño de la muestra

$\mathrm{N}$ : Tamaño de la población finita

$Z \propto$ : Parámetro estadístico que depende del nivel de confianza

$e$ : Error de estimación máximo aceptado

$p$ : Probabilidad que ocurra el evento estudiado (éxito)

$q$ : Probabilidad que no ocurra el evento estudiado (1-p) 
Igualmente, el procedimiento pedagógico se implementa de acuerdo a los contenidos temáticos del plan de asignatura de las materias Gestión del Talento Humano y Creatividad e Innovación en Ingeniería, reconociendo como actores activos del proceso tanto al docente y al alumno, donde ambos se involucran en el diseño de las actividades. La primera fase del proceso metodológico inicia con la revisión y actualización del plan de asignatura, alineándolo a la nueva propuesta de formación por competencias y basado en un modelo desarrollista implementado por la Universidad de la Costa. La figura 2 presenta los elementos que se tendrán en cuenta como componente base para la metodología.

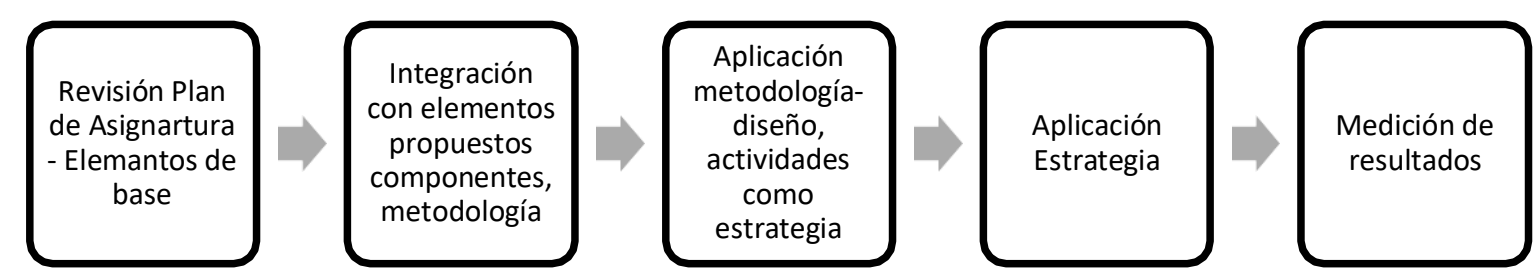

Fig. 1. Fases del proceso metodológico. Fuente: Autores

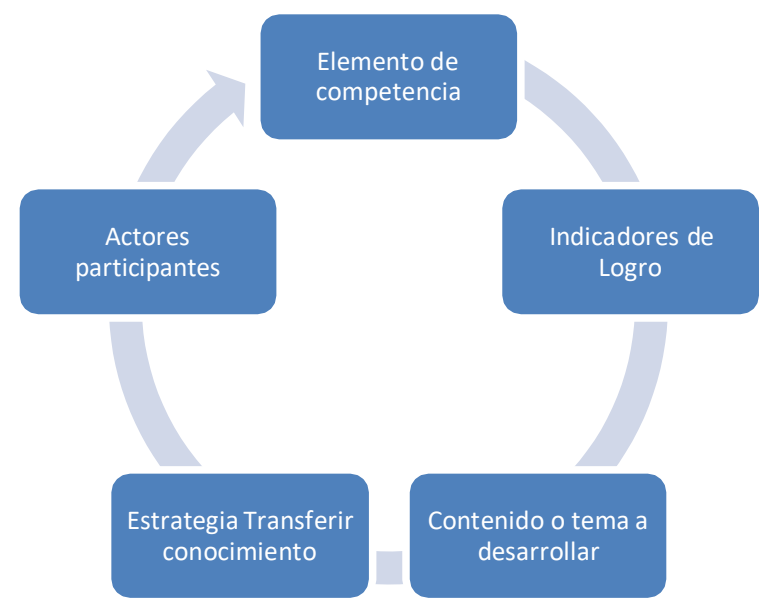

Fig. 2. Base de Metodología. Fuente: Autores

El elemento de competencia es el componente más importante del plan de asignatura, puesto que en él se definen el saber saber, saber hacer y saber ser, que a su vez están ordenados a la misión de la Universidad y al objetivo de esta investigación. Para poder medir el alcance del elemento de competencia es necesario la definición de los indicadores de desempeño, los cuales son componentes que se evalúan por cada temática desarrolladas en las unidades de trabajo. No obstante, para la distribución de estas unidades, es importante utilizar estrategias de trabajo tanto en el salón de clases como en el momento de trabajo independiente, que es el complemento para la apropiación del conocimiento para que se pueda evaluar cada uno de los logros que integran el elemento de competencia.

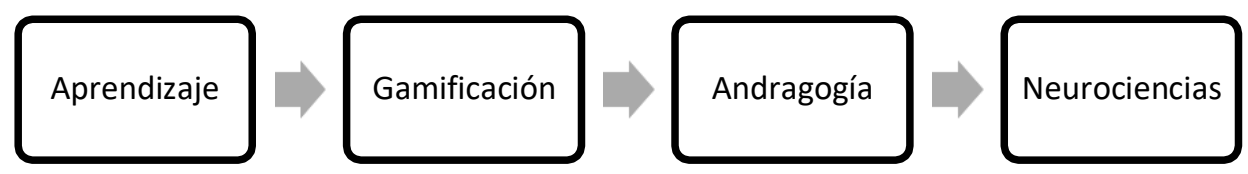

Fig. 3. Componentes de la metodología. Fuente: Autores

La figura 3 describe los componentes de esta propuesta metodológica. Se busca una alternativa pedagógica basada en el aprendizaje divertido, que involucre técnicas y herramientas para adultos, sin importar el orden de interacción porque están conectados entre sí para cada una de las actividades diseñadas que se implementaron. Cuando el aprendizaje está enfocado a una población adulta, [10] se entiende que la andragogía es la disciplina educativa que trata de comprender al adulto psicológico, biológico y social. En esta misma dirección la andragogía es: La ciencia y el arte que, siendo parte de la Antropología y estando inmersa en la educación permanente, se desarrolla a través de una praxis fundamentada en los principios de Participación y Horizontalidad; cuyo proceso, al ser orientado con características sinérgicas por el facilitador del aprendizaje, permite incrementar el pensamiento, la autogestión, la calidad de vida y la creatividad del participante adulto, con el propósito de proporcionarle una oportunidad para que logre su autorrealización [11]. 
En ese sentido, los juegos diseñados en colaboración con los estudiantes, están acompañados de retos en busca del desarrollo de los temas propuestos. La generación de ideas se realiza por medio de comunidades de práctica alineado al plan de asignatura. Se integran todos los elementos con los componentes propuestos.

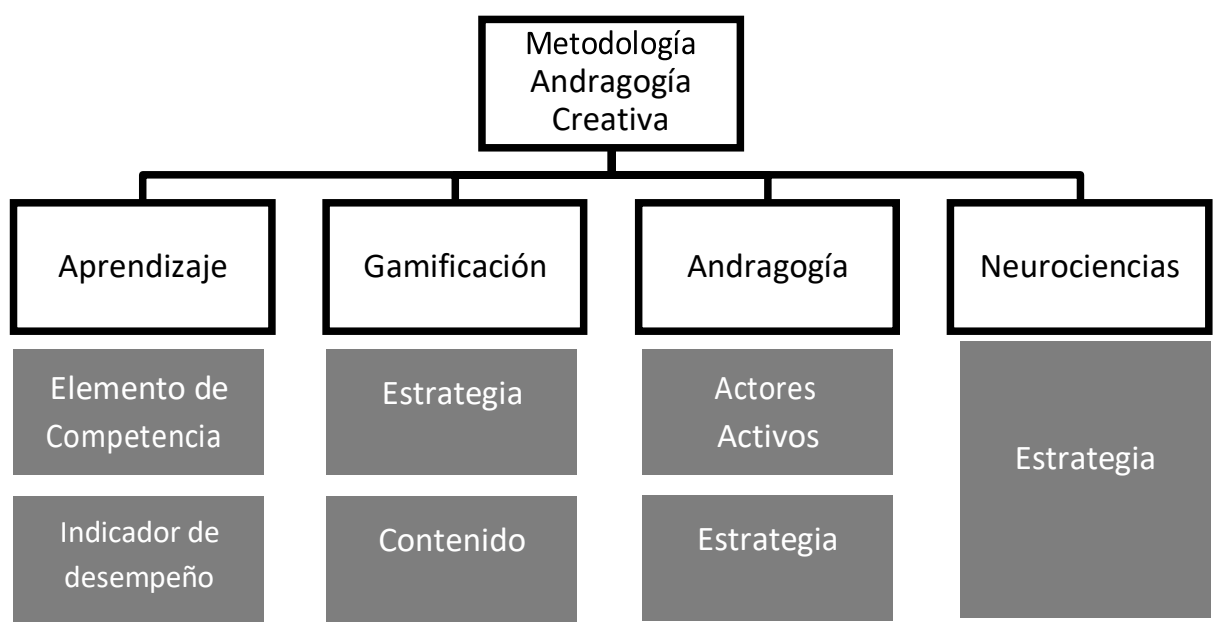

Fig. 4. Integración plan de asignatura-metodología. Fuente: Autores

La figura 4 explica que la estrategia es fundamental en el aseguramiento del conocimiento. Sin embargo, se requiere de una interacción entre todos los componentes para generar empoderamiento entre los participantes que permita generar nuevas propuestas para aplicar dentro del aula, siempre en busca de la autonomía y desarrollo de competencias genéricas y específicas en los futuros Ingenieros industriales.

\section{RESULTADOS}

Los resultados de esta investigación fueron medidos con un instrumento tipo encuesta, para un tamaño de muestra calculado de 65 estudiantes según se sugiere en la metodología de esta investigación. Preliminarmente, se verificó el rango de edades para esta investigación a fin de observar la aplicación de esta propuesta de enseñanza andragógica a la población de adultos jóvenes y adultos en el aula de clases. En este sentido la figura 5 señala que el $69 \%$ de la muestra está en un rango de edad de 17 a 20 años, mientras que el 39\% se encuentra entre los 21 y 30 años de edad.

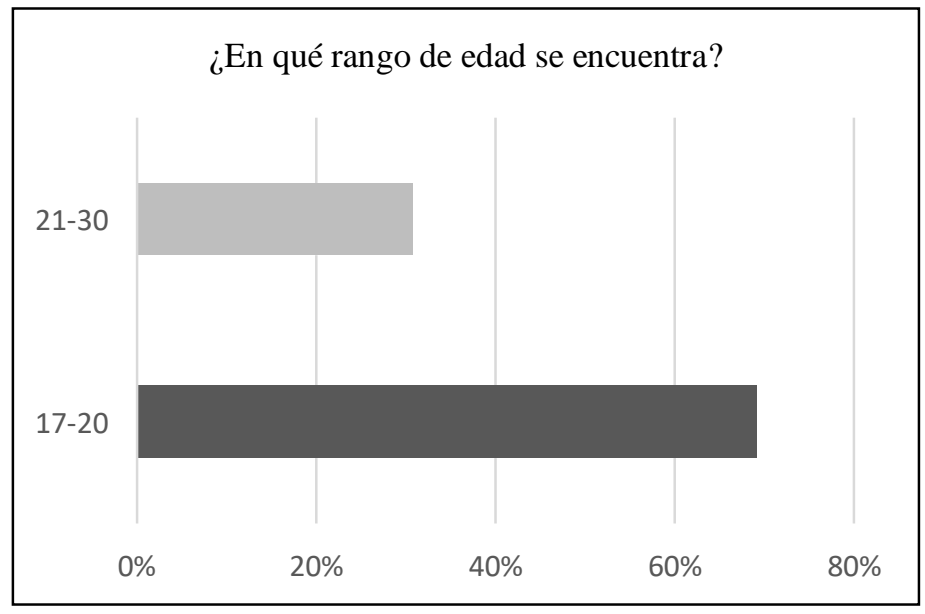

Fig. 5. Rango de edades de esta investigación. Fuente: Autores

Posteriormente se preguntó a este grupo de alumnos si estaban dispuestos a participar activamente en este proceso o si deseaba que el docente realizara su clase magistral para transmitir el conocimiento. Se encontró que el $90 \%$ de las personas encuestadas aceptó participar en esta metodología, como se aprecia en a figura 6. 
BILO Vol. 1 no. 1, Julio- Diciembre de 2019

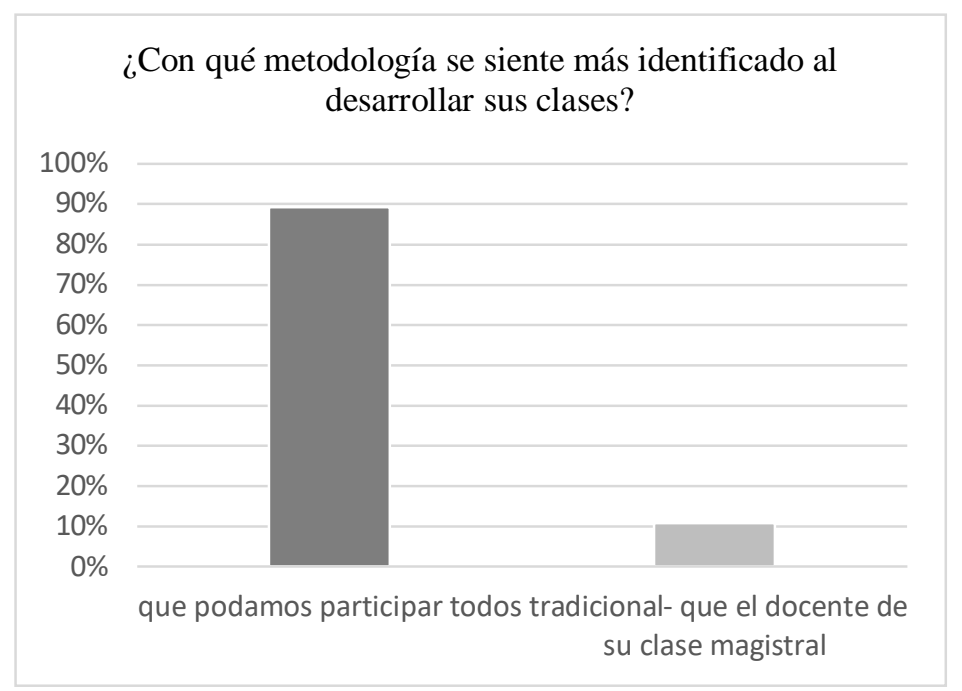

Fig. 6. Resultados a la pregunta de metodología en el aula de clases en la muestra tomada para este estudio.

En el caso de la percepción del estudiante respecto a si la metodología de esta investigación, que trabaja entre sus fases el proceso lúdico como medio de enseñanza a adultos y adultos jóvenes, ayudaba a mejorar su recordación de los temas estudiados o si para él fue un ejercicio desgastante. La figura 6 describe que el $98 \%$ manifestó recordar la temática aplicando las estrategias diseñadas, lo que sugiere una transferencia apropiada de la Gestión del conocimiento con clases experienciales, involucrando a todos los participantes, mientras se va desarrollando el plan de asignatura y los indicadores de logros establecidos por los elementos de competencia de cada unidad.

\section{Que piensas de las clases que se desarrollan con actividades lúdicas?}

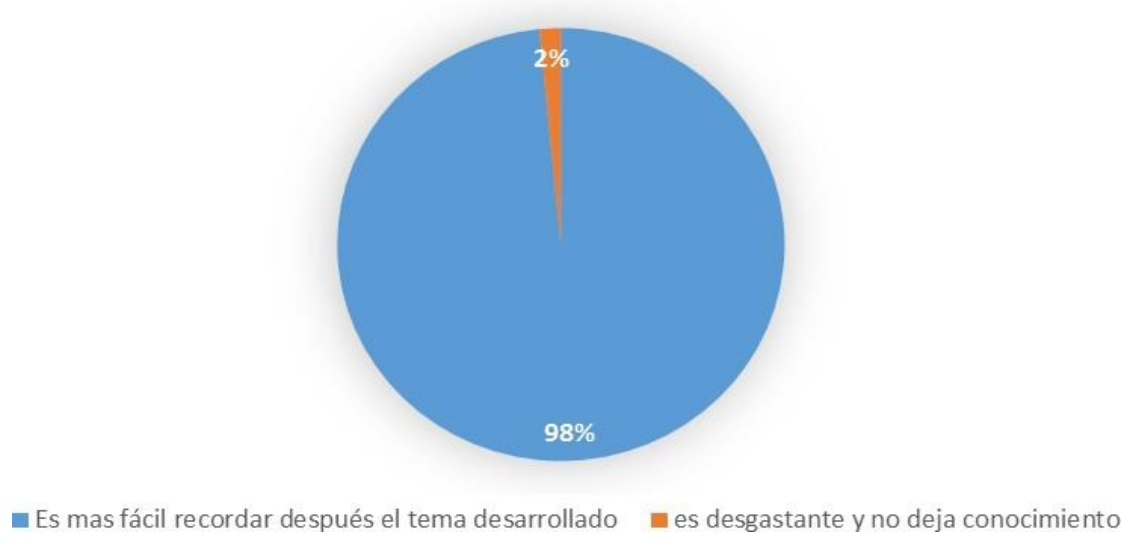

Fig. 7. Resultado encuesta realizada -impacto de clases lúdicas

Las estrategias diseñadas por los estudiantes, son consideradas como resultados de la aplicación de la metodología. Se presentan algunas estrategias que fueron aplicadas por los participantes.

\section{A. Estrategia Tejiendo ideas}

La figura 8 muestra el Desarrollo de esta estrategia. Se separa el aula en dos equipos que competirán entre ellos, se entrega una madeja de lana para cada equipo y previamente deben leer el tema asignado. Con apoyo audiovisual se presentan preguntas y retos sobre el tema.

El participante que sepa la respuesta correcta levanta su mano y contesta, si acierta teje su dedo y el equipo que primero teja a todos sus participantes será el ganador. Cada participante puede participar por una única vez por ronda, comprometiendo así a todos los participantes a ser parte activa del juego. 


\section{B. Estrategia La torre de competencias}

El desarrollo de esta actividad, la cual consiste en tomar el modelo por competencias y realizar una torre que represente todos los pilares que la conforman como un profesional integral. La actividad tiene como objetivo reconocer e identificar las competencias que posee en la actualidad y a partir de ahí generar un plan de acción para cubrir la brecha detectada en sí mismo. La actividad trabaja las competencias del Ser (liderazgo, trabajo en equipo, recursividad, atención al detalle), Saber (manejo de conceptos, teorías y autores sobre el tema de competencias), Hacer (la estructura de la torre).

Igualmente, a los participantes en la encuesta se les preguntó cómo califican de 1 a 5 puntos la clase aplicando la metodología basada en una experiencia y no sólo un contenido o tema presentado de manera magistral dictado por el docente. Al promediar las calificaciones se obtuvo una medida de 4,74. Esto sugiere que la participación del estudiante en su proceso de aprendizaje desde la experiencia en el aula, es apropiada para que los estudiantes gestionen el conocimiento de una forma lúdica, divertida e innovadora mientras se sienten protagonistas y diseñadores de su propio aprendizaje.

\section{Si pudieras elegir la metodología para desarrollar clases, ¿cual prefieres?}

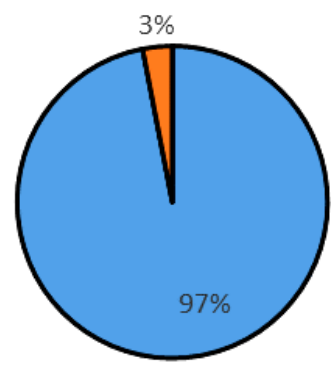

口dinámica y participativa- que todos nos involucremos

口tradicional- que sólo el docente desarrolle la clase

Fig. 8. Resultado encuesta realizada-Preferencia de Metodologías.

El 97\% los participantes encuestados manifestaron que eligen aplicar la metodología en clases que sea participativa y dinámica, generando compromiso y esfuerzo por el cumplimiento de los objetivos de logro, reconociéndolo como un indicador de importancia para el fortalecimiento de las competencias del saber dentro de su profesión como Ingenieros Industriales.

\section{CONCLUSIONES}

Se realizó un estudio de caso a un grupo de adultos jóvenes y adultos en una proporción de $69 \%$ y $31 \%$ respectivamente, para validar la implementación de la metodología propuesta "andragogía creativa" donde el 90\% de los encuestados se mostró dispuesto a participar en esta alternativa de enseñanza en una escuela de ingeniería industrial, de una universidad en Colombia. Con los resultados de esta investigación, se encontró que el 98\% de los estudiantes encuestados se halló más predispuesto a recordar de una forma más fácil los temas estudiados desde actividades lúdicas que por otro tipo de técnica.En este sentido, se cree que este modelo es apropiado para desarrollar y transferir el conocimiento a nivel educativo superior, siempre y cuando se trace el cumplimiento de los objetivos propuestos desde los elementos de competencia de la asignatura. La temática desarrollada es innovadora y de práctica adaptación para todos los participantes involucrados, permitiendo gestionar el conocimiento por medio de técnicas gamificables y estrategias diseñadas para el desarrollo de las competencias requeridas del ser y el saber, convirtiendo el aprendizaje de un proceso convencional y tradicional a un aprendizaje creativo y divertido direccionado a la población adulta.

Los ambientes elegidos y diseñados para el desarrollo de las actividades fueron los apropiados para activar el aprendizaje basado en las emociones y la práctica vivencial, convirtiendo al estudiante como protagonista y responsable de gestionar su propio conocimiento. Se cree que el aprendizaje ha evolucionado y dentro de las aulas de clases se pueden instar experiencias que involucren a los estudiantes con sus emociones y diferentes estilos de gestionar el conocimiento. 
BILO Vol. 1 no. 1, Julio- Diciembre de 2019

Se espera para futuros trabajos, la inclusión de esta metodología en espacios que no solo involucren a una facultad en específico sino que se evalúe desde un proceso interdisciplinar y con varios semestres en el estudio, para estimar qué tanto influyen factores como el tipo de carrera, la proporción en el rango de edades, la cantidad de estudiantes evaluados y las asignaturas analizadas, sobre la efectividad de esta propuesta de enseñanza por competencias desde la perspectiva de la gestión del conocimiento.

\section{REFERENCIAS}

[1] V. López., C. Arias. y K. Restrepo., Un estudio de la relación entre la empatía y la creatividad en alumnos, Revista Complutense de Educación, pp. 1133-1149, 2017.

[2] Suárez, O. (2016). Aproximación al origen de la noción de objeto de aprendizaje: revisión histórico bibliográfica. INGE CUC, 12(2), 26-40. https://doi.org/10.17981/ingecuc.12.2.2016.03

[3] M. Pierella, La autoridad profesoral en la universidad contemporánea, Perfiles Educativos, pp. 140-156, 2014.

[4] A. Marczewski., Gamification User Types, 2013.

[5] G. Zichermann, Beyond the hype: 5 ways that big companies are using gamification. GIFAOM., 2013.

[6] G. Velategui, Gestión de aula y gamificación, 2015.

[7] J. Margolis. y M. knowles., The modern practice of adult education, Train Dev., 1970.

[8] D. R. Ripoll, Neurociencia Cognitiva, 2014.

[9] J. Cumpa, Neurociencia Cognitiva y Educación, 2004.

[10] C. Santos., Andragogía: Aprendiendo a enseñar adultos, Resende, RJ, 2010.

[11] M. Bartle, Andragogy - Research Starters Education, 2014. 